\title{
La Nueva Teoría Estratégica (NTE) de la Comunicación como modelo para la construcción de una estrategia de Vinculación con el Medio (VCM) en dos instituciones de educación superior del sur de Chile
}

\author{
doi: 10.33264/rpa.202001-06 \\ Jorge Pinilla Andrade \\ Antropólogo, Universidad Austral de Chile \\ Patricia Ferrada Montecinos \\ Académica, Universidad Adventista de Chile
}

\section{Resumen}

En este artículo se analizan y comparan las estrategias de Vinculación con el Medio (VcM) de dos universidades del sur de Chile: Inacap sede Coyhaique y Universidad del Bío Bío. El acercamiento al objeto de estudio se realiza desde el enfoque de la Nueva Teoría Estratégica (NTE) de la Comunicación, que revela aprendizajes significativos y que contribuye a optimizar tanto las áreas de VcM como de comunicaciones al interior de las Instituciones de Educación Superior (IES) bajo estudio. Preliminarmente hay un trabajo que profundiza y aclara aspectos que, a la luz de la Nueva Teoría Estratégica de la Comunicación, se aplican al estudio comparativo, para finalizar con los principales hallazgos y conclusiones.

Palabras claves: Nueva Teoría Estratégica de la Comunicación, comunicación estratégica, vinculación con el medio, tercera misión, universidades

\section{Abstract}

This article analyses and compare the strategic university engagment of two universities from south of Chile: Inacap campus Coyhaique and Bio Bio university. The approach to the object of study is trough the New Strategic Theory of Comunication who shows learnings to promote improvements either on the university engagement and comunications fields, within high education institutions. Before the article deepens and clarifies some concepts that, from the point of view of the New Strategic Theory of Comunication, apply to the comparative study. Finally the article shows the mains finding and conclusions.

Keywords: New Strategic Theory of Comunication, strategic comunication, university engagement, third mission, universities 


\section{Introducción}

El siguiente artículo revisa las políticas y acciones de Vinculación con el Medio (VcM) de dos universidades del sur de Chile, desde una mirada conceptual enmarcada en la Nueva Teoría Estratégica (NTE) de la Comunicación. La investigación plantea preguntas respecto a si existen mayores coincidencias o discrepancias en el desarrollo de las áreas de VcM en ambas instituciones. La problematización nace fundamentalmente de la pregunta ¿cuáles serán las variables más relevantes de la NTE que sirven para apalancar la VcM desde la Comunicación? A partir de aquí se plantea la hipótesis: "La Nueva Teoría Estratégica (NTE) de la Comunicación sirve como modelo y base para una gestión catalizadora de la Vinculación con el Medio en las instituciones de educación superior, específicamente para las instituciones bajo análisis en el presente estudio".

En primer lugar, se lleva a cabo una depuración conceptual en la cual se revisan las acepciones más aceptadas por la teoría para acordar qué se entenderá por VcM, tanto desde la mirada interna de las instituciones, como del enfoque gubernamental y de segmentos gravitantes relacionados con la VcM, incorporando además una perspectiva histórica del concepto y de la comunicación estratégica. Para el desarrollo de esta fase, además del trabajo de gabinete, se desarrolló un acercamiento en terreno, bajo un marco de investigación cualitativa, que permitió obtener información de primera fuente.

De acuerdo a herramientas obtenidas desde la NTE de la Comunicación, el estudio propone un esquema de análisis en base a atributos centrales y efectivos al momento de describir el fenómeno, comparar sus elementos y generar síntesis de información. Con los logros del estudio se vislumbran alternativas de planificación y acción ante desafíos apremiantes en el área de VcM (como un aspecto obligatorio de acreditación de las instituciones de educación superior en Chile a partir del año 2020), así como postular la necesaria complementación entre comunicación estratégica y el área de VcM, para una exitosa labor sinérgica entre las universidades y el territorio donde se emplazan.

\section{Definición de conceptos}

\section{Comunicación}

El presente trabajo coincide con Claudia Salas (2011:234) en que la comunicación es una ciencia multidisciplinar y transdisciplinar. Esto porque el proceso de comunicación es un proceso que se da entre grupos humanos y por eso, la teoría de la comunicación y el propio concepto de comunicación se basa en un fundamento antropológico, tendrá que reflejar el concepto de hombre que se tenga (Martínez Fresneda, 2006:17).

El desarrollo de la teoría de la comunicación ha oscilado desde enfoques interaccionistas, simbólicos, interpretativos y de acción comunicativa, donde las corrientes de pensamiento se nutren de valiosas miradas como las provenientes de 
la semiótica del discurso, la semiótica cognitiva del discurso y las ciencias cognitivas, entre otras. En este desarrollo ha habido amplitud de espacio para establecer sinergias y puntos en común con áreas como las relaciones públicas en general, el management y la reputación de marca, así como también con ámbitos más cercanos al marketing, tal como lo es el branding. A grosso modo, las corrientes teóricas han seguido diversas escuelas de pensadores y filósofos, y han dialogado fecundamente con disciplinas sociales como la sociología y la psicología. A este respecto Herrera y Bendezú (2017:136) señalan que la comunicación estratégica es hoy el paradigma en cuanto lograr ordenar íntegramente habilidades tras un propósito, en un escenario de complejidad -pues incorpora elementos racionales, emocionales y experienciales, y es esencialmente la dimensión aplicativa de las diversas ciencias de la comunicación: "Por tanto, si la comunicación implica actividades de mutua influencia, de intercambio, de persuasión, de difusión y de intervención organizacional, esta es estratégica" (139).

\section{Comunicación estratégica}

La estrategia puede entenderse como el modo de actuar en una multiplicidad de campos y en cualquier grupo que tenga un objetivo común y que para alcanzarlo se alinee metodológicamente tras dicho propósito. La estrategia permite alcanzar un fin y tradicionalmente se la asocia al ámbito militar (Cambria, 2016:1). Ahora bien, la diferenciación entre comunicación y comunicación estratégica, estriba en que esta última es una superación de la primera, y que, en cierta medida, transforma el pensamiento de la comunicación (Salas, 2011:235), lo complejiza, pero al mismo tiempo descarta elementos innecesarios y métodos inapropiados, para pasar del reduccionismo y la fragmentación analítica de la realidad a la asunción del pensamiento complejo (Martínez, 2015:723). La comunicación y la estrategia se relacionan y surge entonces la comunicación estratégica, apoyándose mutuamente, fortaleciendo su accionar. Incluso antes de nominarla como tal, antes de estudiarla, funcionaban exitosamente de la mano, comunicación y estrategia (Pérez, 2014:18). Aun cuando existen diversas miradas teóricas sobre la definición y los alcances de la comunicación estratégica (Nothhaft, 2016:71) este trabajo adscribe a que la comunicación estratégica agrega al propio acto natural de comunicar la incorporación del necesario entendimiento entre los actores presentes en el medio donde se entabla la comunicación, quienes a nivel interpersonal configuran un cuadro racional, mientras que a modo colectivo entablan un cuadro relacional con el medio (Pérez, 2012:138; Salas, 2011:234). Además, en la comunicación estratégica, los contenidos se elaboran desde un sustrato que intenciona comportamientos en las audiencias, sean estas externas o internas (Grunig et al, 2015:11), donde los distintos participantes o stakeholders influyen también generando un campo de mutuos intereses en juego (Pérez, 2008). En este sentido la comunicación estratégica aporta con elementos innovadores en el campo práctico, contribuyendo a la generación de instrumentos metodológicos que permiten valorar analíticamente el estado, avances y desarrollo de los procesos comunicacionales, considerando la fluidez y complejidad del fenómeno en su multidimensionalidad (Massoni et al, 2015:90). 


\section{La Nueva Teoría Estratégica (NTE)}

Tras la búsqueda de un nuevo paradigma que entienda la estrategia y la comunicación más allá del management, Rafael Alberto Pérez plantea en su libro Estrategias de comunicación: "Necesitamos una teoría estratégica menos geométrica y más hermenéutica, menos racional y más relacional” (Pérez 2014:20, Herrera \& Bendezú, 2017:145). Nace entonces, la NTE o Nueva Teoría Estratégica. El núcleo de construcción significativa de la comunicación, perspectiva adoptada en el desarrollo de este trabajo, se encuentra en el estrategar, donde cumple un papel clave la intersubjetividad de los participantes con un código común. Es en este escenario donde "van a ser los contextos en los cuales se encuentran los participantes de la comunicación los que van a poder regular la confianza de la información comunicada" (144), donde además "la comunicación no está dada de antemano o entregada plena o en dosis, sino que se va construyendo en una actividad coordinada" (145). La importancia de la nueva teoría estratégica radica en que "incorpora a la interpretación como una condición humana. Todos interpretamos mensajes desde los puntos de vista cognitivo y emotivo. Las palabras, y los diferentes signos, contienen un significado interpretativo que, en sí mismo, implica una carga racional y a la vez emotiva" (148).

La relevancia fundamental de la Nueva Teoría Estratégica de la Comunicación radica en su giro epistemológico que coloca al ser relacional en el centro de la teoría (Arribas, 2017:70), piedra angular y "principio relacional y humanizante de la estrategia para la NTE” (Herrera \& Bendezú, 2017:152).

\section{Marco normativo para la acreditación de universidades en Chile.}

A partir del año 2006 se estableció en Chile un sistema nacional de aseguramiento de la calidad de la educación superior a través de la Ley $\mathrm{N}^{\circ} 20.129$, promulgada por la Presidencia de la República en octubre y publicada en el Diario Oficial el 17 de noviembre del mismo año. Mediante dicho cuerpo legal se creó la Comisión Nacional de Acreditación CNA, "organismo autónomo que gozará de personalidad jurídica y patrimonio propio cuya función será evaluar, acreditar y promover la calidad de las Universidades, Institutos Profesionales y Centros de Formación Técnica autónomos y de las carreras y programas que ellos ofrecen" (Ley $\mathrm{N}^{\circ} 20.129,2006$ ). Una de las principales tareas especificadas en esta Ley fue la acreditación de las entidades de educación superior, proceso mediante el cual una agencia legalmente autorizada, otorga reconocimiento público a una institución académica o de investigación porque reúne ciertos estándares y cualidades previamente establecidas (Pineda de Alcazar, 2006:145). Para el cumplimiento de la normativa, se designó a la CNA como el ente encargado de elaborar y establecer los criterios y estándares de calidad para la acreditación institucional, la cual se definió voluntaria para los organismos educacionales interesados, los cuales deberían acreditarse de forma obligatoria en docencia de pregrado y gestión institucional, y optativa para las áreas de investigación, docencia de postgrado y VcM. 


\section{Vinculación con el medio}

Hay un énfasis expresado por Von Baer (2009:469) donde señala que las instituciones de educación superior son un agente insustituible para el desarrollo de sus respectivas regiones o comunidades beneficiadas (Clark et al, 2016:1). En un contexto contemporáneo, se han superado las dos clásicas orientaciones de enseñanza e investigación, dando paso a una "tercera misión" (Barker, 2015:477), relacionada con potenciar habilidades y facultades que fortalezcan el territorio donde se encuentran insertas (Loi 2015:855; Secundo et al, 2017:229), y decididamente contribuyan al repunte económico de dichos territorios (Clark et al, 498). Los nuevos objetivos por parte de las universidades y que se entroncan con su labor de VcM, son: ofrecer educación continua a poblaciones regionales, retener población, requerir de servicios, proporcionar infraestructura, aportar liderazgo de elite, generar conocimiento al servicio de la región, apoyar con asesorías para el desarrollo regional, ser empleadores significativos en las regiones (Schubert \& Kroll, 2016:468), entre otros.

La VcM es vista como la tercera función esencial de las universidades, de valor equivalente a la docencia y la investigación, y necesaria para nutrir y retroalimentar la calidad y pertinencia de éstas (Von Baer, 2009:467). Sin embargo, siguiendo a Dougnac (2016), a pesar que ha existido una propagación inaudita del desarrollo de VcM, ésta área carece de una profundización teórica adyacente que le asigne mayor respaldo y ordene su desarrollo hacia propósitos claramente definidos. En este sentido, ha existido un notable desarrollo a nivel de políticas institucionales " $y$ aunque ha generado creciente interés en distintas instancias de discusión, ha sido escasamente acompañado de una revisión crítica de estos conceptos a nivel de publicaciones científicas" (2). A esto debemos sumar los obstáculos endémicos para la divulgación de los resultados en las ciencias sociales y de la comunicación en idioma español (Mancinas et al, 2016:241), cuestión también preocupante para esta área del conocimiento, debido a que afecta directamente la socialización de sus resultados y de posibles propuestas eficaces que contribuyan a resolver las diversas problemáticas.

La forma como las entidades de educación superior han entendido el concepto de VcM ha transitado desde una perspectiva unidireccional en la cual las instituciones desarrollan una serie de actividades como la extensión hacia la comunidad que las circunda (Merino, 2004:77), hasta un enfoque bidireccional en el cual se entiende la idea de "vinculación" como el eje principal de las acciones que lo insertan con la comunidad y que involucra a los stakeholders que participan de su desarrollo (Von Baer, 2009: 471). En este sentido la conceptualización de la idea de VcM debería incorporar a todos los stakeholders que se relacionan con la institución, pero también, las diferentes dimensiones que abordan las entidades de educación superior, más allá de la docencia o la investigación (Fleet et al, 2017:36) .

Hay autores que expresan la relación íntima entre vinculación con el medio y otras características emergentes propias de las universidades, en el contexto contemporáneo de las organizaciones. Un ejemplo es la cristalización de las nociones 
fundamentales de la institución universitaria en su sello de compromiso social (Beltrán-Llevador et al, 2014:5) universitario, en donde se han incorporado importantes elementos de la Responsabilidad Social y del Valor Compartido. En lo sustancial, se trata del mismo enfoque de gestión organizacional (Ruff \& Ruiz, 2012:264) y lo que incorporan son aspectos más relevantes en las funciones de las universidades, que dice relación con impulsar políticas que fomenten el emprendimiento, la innovación y la cooperación social, en un amplio abanico de temáticas.

\section{Metodología}

La metodología empleada para esta investigación es de tipo descriptiva, transversal, con un enfoque metodológico aplicado. Las técnicas de investigación correspondieron al tipo cualitativo, específicamente entrevistas semi-estructuradas a informantes claves y expertos.

\section{Objeto de estudio}

Correspondió a dos instituciones de educación superior del sur de Chile: Universidad INACAP sede Coyhaique y Universidad del Bío-Bío sede Chillán. Ambas entidades corresponden a instituciones de educación superior reconocidas por el Estado y acreditas por la Comisión Nacional de Acreditación (CNA-Chile). Inacap es una corporación de derecho privado, fundada el 21 de octubre de 1966, se define como un sistema integrado de educación superior formado por el Centro de Formación Técnica, Instituto Profesional y Universidad, sumando más de 120.000 alumnos, con 77 carreras técnicas y profesionales de pregrado. Cuenta con 26 Sedes, en 16 regiones y con $316.000 \mathrm{Mts}^{2}$ construidos de infraestructura. Al momento de la investigación, Universidad Inacap se encontraba acreditada por la CNA por 2 años, el CFT por 7 años y el Instituto Profesional por 6 años.

La Universidad del Bío-Bío es una institución estatal y regional, perteneciente al Consejo de Rectores de las Universidades Chilenas ( $C R U C H)$. Su origen se remonta a la Universidad Técnica del Estado, UTE en Concepción y la sede Chillán de la Universidad de Chile, las que constituyen las dos sedes que la conforman. Posee 6 facultades y 10 mil estudiantes de pregrado, 39 carreras o programas de pregrado, 2 programas de doctorado y 21 programas de magíster, aproximadamente 550 académicos y $582.830 \mathrm{Mts}^{2}$ de infraestructura. Actualmente se encuentra acreditada por CNA-Chile por un período de 5 años.

\section{Métodos y técnicas de investigación}

La investigación se apoyó en técnicas de investigación basadas en entrevistas semiestructuradas a tres grandes grupos de informantes claves:

- Grupo 1: Profesionales a cargo de VcM.

- Grupo 2: Panel de expertos.

- Grupo 3: Actores claves al interior de las instituciones bajo estudio. 


\section{Entrevistas semi-estructuradas}

Se optó por un instrumento de preguntas abiertas, que permitiera indagar en dos grandes áreas de interés o dimensiones: Nueva Teoría Estratégica (NTE) de la Comunicación y VcM, las cuales a su vez se desglosaron en cinco y cuatro sub-áreas o variables, respectivamente. El instrumento fue validado por juicio de expertos.

- Nueva Teoría Estratégica (NTE) de la Comunicación: Las comunicaciones, futuribles, visión del ser humano, escucha y diálogo con las audiencias, aspecto emocional.

- VcM: Desarrollo regional, políticas estatales, sistematicidad y prospección, medición de impacto.

\section{Definición de las variables}

- Comunicaciones: Tiene relación con las áreas de comunicaciones o de comunicación estratégica en las instituciones.

- Futurible: Espacio simbólico que se pretende ocupar en las audiencias identificadas como estratégicas. Es lo que se pretende que piensen, sientan y digan de la marca o institución.

- Visión del ser humano: Esta variable, coloca a la persona en el centro de las acciones y reflexiones y le otorga la facultad relacional, contraponiéndose a una perspectiva puramente racional en la cual el énfasis está puesto en las instituciones y/o en las acciones.

- Escucha y diálogo con las audiencias: Se refiere al espacio deliberadamente asignado para establecer una relación fluida entre las instituciones con sus audiencias estratégicas.

- Aspecto emocional: Dice relación con el establecimiento de las relaciones humanas, relaciones que se fundan desde la emoción, vale decir, se asocian a qué emerge al interior de cada persona al enfrentarse a una marca/institución.

- Desarrollo local/regional: Se refiere a las construcciones racionales/lingüísticas expresadas en torno a la valorización sobre el objeto de estudio. Cómo se inserta con su entorno, cómo aporta a su desarrollo y del mismo modo, cómo se beneficia de él.

- Políticas estatales: Se refiere a la percepción en torno a la macropolítica atingente al ámbito de la educación, incluida en ella los aspectos relativos a la promoción de la VcM por parte del Estado.

- Sistematicidad y Prospección: Se refiere al sentido que tienen las acciones o políticas internas de VcM en las instituciones, a cómo se construyen, articulan, interactúan y cómo se proyectan en el tiempo.

- Medición de impacto: Relativo a los mecanismos por los cuales las instituciones de educación superior (específicamente en sus áreas de VcM) abordan sus resultados en vista de una mejora continua. 


\section{Desarrollo}

El análisis de los resultados se efectuó con base en los hallazgos, los cuales fueron organizados de acuerdo a las dimensiones y variables establecidas. La siguiente información es el resultado del cruce de variables:

Comunicaciones: Para los expertos hay coincidencia en que las áreas de comunicación y de VcM deben trabajar en conjunto, inclusive se propone que Comunicaciones incorpore a VcM como un apéndice de ésta. Sin embargo, en las dos instituciones estudiadas esto no fue así, en la Universidad del Bío Bío, VcM está bajo el alero de la Dirección de Relaciones Institucionales y comunicaciones colabora con ellos en la difusión. En Inacap, actúa como una vicerrectoría, la cual declara trabajar de forma articulada con comunicaciones, docencia e investigación. Existe desconocimiento, tanto en las audiencias internas y externas, sobre qué es VcM y, por ende, se subestima su importancia. Se identifica una falencia en la comunicación de sus actividades y propósitos. La preocupación por contar el quehacer de la institución que es amplio e importante-, parece dejar de lado la trascendencia de las acciones de vinculación, que a veces son menos mediáticas.

Futuribles: La acción de VcM es estratégica y puede potenciar a las instituciones de educación superior otorgándoles un rol protagónico incluso a nivel país, si las instituciones usaran esta instancia más allá de las capacitaciones, seminarios o hitos que permiten relacionarse con el entorno. En Inacap, el futurible esperado de VcM se nutre en que su imagen/referencia esté asociada a su contribución a través de formación continua, presentaciones de arte o propedéuticos, aunando aspectos como desarrollo regional y local, que se ven potenciados con propuestas en que articulan las áreas de docencia e investigación para generar proyectos que, para que sean realmente trascendentes, deberían ser a largo plazo y sistemáticos. En la Universidad del Bío Bío las líneas de desarrollo son amplias pero dispersas, por lo que la articulación se ve más difusa, aunque con seguridad se consigue probablemente a costo de mucho más trabajo.

Visión del ser humano: Aunque existe una tendencia natural a colocar el rol del ser humano en el centro de la política de VcM y de las comunicaciones estratégicas, hay cierta dificultad en explicar detalladamente cómo se materializa. Se aprecia una respuesta intuitiva pero no racionalizada detalladamente. La institución debe ser capaz de conocer quiénes son las personas que le interesan y para qué le interesan. Los expertos en comunicación proponen definir los públicos, más allá de la categoría interno/externo, como ciudadanos, como personas, quiénes son y cómo gestionar las relaciones con ellos.

Escucha y diálogo con las audiencias: En Inacap hay una tensión entre lo que a nivel central indican se debe desarrollar en materia de VcM, donde cuentan con amplio margen de actuación, según los informantes; sin embargo, esto también implica saber leer e interpretar adecuadamente las exigencias y expectativas de la comunidad, cuestión para la cual no se cuenta con instrumentos de medición sistemática, que revelen eficiencia y resultados. Para el caso la Universidad del Bío Bío 
un aspecto a subrayar es su posición dentro de un enorme ecosistema de organizaciones que despliegan VcM, para la cual la estrategia y la implementación de una adecuada escucha de audiencias se hace imperativa. En ambos casos, el llamado es a co-construir con los públicos internos y externos. Es evidente, que cada estamento tiene una concepción distinta de lo que es VcM. Para los funcionarios, es más social, cercana al entorno próximo. Para los estudiantes es una mirada más estratégica hacia lo que realmente les aporte en su formación y desarrollo. Hay necesidades de vinculación distintas según el estamento.

El aspecto emocional: Lo emocional, al igual que la visión del ser humano, se aprecia como un aspecto marcadamente desconocido, y que actúa, pero de forma no sistemática ni planificada. Se acusa una necesidad por trabajar los mensajes, para llegar en forma directa a sus audiencias, con atributos claves de las organizaciones como solidaridad y compromiso, materializadas en acciones.

Desarrollo local/regional: Al tratarse de dos universidades regionales, este punto es clave y compartido, en cuanto enfatiza la relación estrecha y sinérgica entre las instituciones de educación superior y su territorio, complementándose, involucrándose mutuamente, en aspectos económicos y sociales. Sin embargo, es importante, a juicio de los expertos, que la política de VcM integre una visión macro, que se proponga abiertamente qué quiere ofrecer al país y cómo quiere ser conocida.

Políticas públicas: Existen coincidencias, respecto a que la libre interpretación del marco normativo, deja un espacio nocivamente abierto a realizar una labor poco rigurosa y sin mayores beneficios, tanto para las organizaciones educativas, como para la comunidad circundante. A pesar de esto, se consigna que la política pública actual respecto a estas temáticas refleja los esfuerzos y el compromiso de la institucionalidad, por potenciar el área y cumplir con las directrices establecidas.

Sistematización y prospección: Existe cierta incertidumbre en la dirección que tomará la VcM en el mediano plazo, aun cuando parece claro que las vicerrectorías de esta área serán la nomenclatura donde se albergarán las acciones e iniciativas. Las funciones específicas que cumplan son la gran interrogante, los que de acuerdo a los informantes transitan entre la articulación, promoción y difusión de las iniciativas.

Medición del impacto: También se distinguen diversos enfoques y miradas, incluso desde puntos bases donde comenzar la discusión. Esto se aprecia, por ejemplo, en el uso cotidiano del concepto de medición de impacto, término ampliamente criticado por autores debido a los significados utilitaristas que arrastran. Para ellos, entre quienes se puede mencionar a Nicolás Fleet, es más apropiado hablar de medición de efectos de la VcM. Instituciones como la Universidad del Bío Bío, reclaman indicadores más precisos a la hora de evaluar, que consignen claramente, por ejemplo, el número de publicaciones, asociaciones o metas que se deberían alcanzar para lograr esta dimensión. 


\section{Conclusiones}

Ante la pregunta ¿Cómo la NTE de la comunicación sirve de modelo y base para una gestión catalizadora de la VcM en las instituciones de educación superior, específicamente para las IES bajo análisis en el presente estudio?, y a partir de la cual se analizaron las acciones y políticas de VcM de las dos entidades estudiadas, podemos generar una serie de recomendaciones, que, a la luz de la NTE de la Comunicación, responden al cómo gestionar de forma eficiente y efectiva el vínculo de las instituciones con su entorno, para desarrollar la "tercera misión": el compromiso de la universidad con su sociedad y con su tiempo.

De acuerdo a las dimensiones analizadas, proponemos las siguientes recomendaciones que permiten gestionar la VcM desde la comunicación estratégica y específicamente, desde la NTE de la Comunicación:

Comunicación como eje estratégico: Las instituciones de educación superior deben contemplar para las áreas de VcM, a la comunicación como un eje estratégico. Que la acción de comunicaciones implique una gestión estratégica que la relacione con los diferentes públicos, que se preocupe de la reputación, de la comunicación digital y sobre todo de informar permanente y profundamente.

La extensión como vínculo dialogante con las personas: El quehacer artístico, cultural no se puede relegar. Junto a las comunicaciones generan las condiciones para que el medio externo confíe en las instituciones; permiten el vínculo dialogante de interacción con la comunidad.

Co-construcción: Se debe ampliar la mirada a la hora de diseñar las políticas y acciones de vinculación, tomando en cuenta las demandas y requerimientos que plantea el entorno, la sociedad civil, las empresas locales y la industria nacional. También se deben considerar los actores internos, los estamentos que conforman la institución, cuyos intereses debieran estar mapeados, identificados y caracterizados en cuanto al tipo de relacionamiento y los objetivos que se espera alcanzar con cada uno de ellos.

Generación compartida de conocimiento: Articular las iniciativas que se ejecutan desde las facultades, desde los estudiantes, las direcciones de desarrollo, formación permanente o transferencia. $Y$ junto con ello promover dichas acciones y difundirlas sistemáticamente, para que sea ampliamente reconocida. Que la productividad se cuente a la comunidad y que desde ahí regrese a la Universidad.

Reflexionar permanentemente en torno al "¿cómo nos ven?": Esto implica escuchar y conversar de forma activa y sistemática con los públicos para conocer cómo perciben ellos a la institución y qué tan efectivo son los aportes que desarrolla a nivel local, regional y nacional.

Perspectiva macro: Es importante reafirmar la inmensidad y diversidad de acciones que se realizan bajo la denominación de VcM, las cuales cruzan desde la clásica extensión cultural y artística, hasta las más tradicionales metas comerciales de generar patentes de innovación, incluyendo las acciones de capacitación, conforme 
las necesidades regionales, desde donde se trabaja con ese impulso originario y se demuestra que ese atributo se vuelve unificador frente a la gran variedad.

Nuevos escenarios, nuevas demandas: La definición de las políticas y acciones de VcM deben estar orientadas a satisfacer las nuevas demandas que exigen los cambios sociales, tecnológicos, culturales, etc. No puede haber un desfase entre la Universidad y las exigencias del entorno (ejemplo, demandas feministas, estallido social, Agenda 2030), debe retroalimentarse permanentemente con una perspectiva global que refleje hacia dónde va el país, la región, la Universidad.

\section{Referencias}

Arribas, A. (2017). Un cambio en el sujeto: la transformación del hombre racional a hombre relacional en un sistema orgánico y aprendiente. En Arribas, A., Herrera Raúl \& Pérez, R.A. (Coord.) Repensando la Estrategia desde la Comunicación, Nueva Teoría Estratégica (pp. 34-69). Universidad de los Hemisferios.

Barker, K. (2015). The challenging Australian policy context for university engagement. Journal of Higher Education Policy and Management, 37(5), 477-489.

Beltrán-Llevador, J., Íñigo-Bajo, E. \& Mata-Segreda, A. (2014). La responsabilidad social universitaria, el reto de su construcción permanente. Revista lberoamericana de Educación Superior, 5(14), 3-18. https://dx.doi.org/10.1016/s2007-2872(14)70297-5

Cambria, A. (2016). La importancia de la comunicación estratégica. Boletín del Instituto español de estudios estratégicos, 42, 1-13. En: https://dialnet.unirioja.es/descarga/articulo/5998262.pdf

Clark, G., Russell, J., Enyeart, P., Gracia, B., Wessel, A., Jarmoskaite, I., Polioudakis, D., Stuart, Y., Gonzalez, T., MacKrell, A., Rodenbusch, S., Stovall, G., Beckham, J., Montgomery, M., Tasneem, T., Jones, J., Simmons, S., \& Roux, S. (2016). Science Educational Outreach Programs The Benefit Students and Scientists. PLoS Biology, 14(2), 1-8. https://dx.doi.org/10.1371/journal.pbio.1002368

Dougnac, P. (2016). Una revisión del concepto anglosajón public engagement y su equivalencia funcional a los de extensión y vinculación con el medio. Pensamiento Educativo. Revista de Investigación Educacional Latinoamericana, 53(2), 1-19

Fleet, N., Victorero, P., Lagos, F., Montiel, B. \& Cutipa, J. (2017). Cuaderno N6 Midiendo la vinculación de las instituciones de educación superior con el medio y su impacto. Estudio de las mejores prácticas en el mundo y desarrollo de instrumento piloto para instituciones chilenas. Santiago: Comisión Nacional de Acreditación CNA. 
Grunig, J., Grunig, Larissa A.\& Aparecida Ferrari, María (2015). Perspectivas de las Relaciones Públicas: resultados del Excellence Study para la comunicación en las organizaciones. Revista Mediterránea de Comunicación, 6(2), 9-28.

Herrera, R. \& Bendezú, R. (2017). La estrategia desde una matriz comunicacional. En Arribas, A., Herrera Raúl \& Pérez, R.A. (Coord.) Repensando la Estrategia desde la Comunicación, Nueva Teoría Estratégica (pp. 135-167) Quito, Ecuador. Universidad de los Hemisferios.

Ley $\mathrm{N}^{\circ}$ 20.129. Diario Oficial de la República de Chile, Santiago, Chile, 17 de noviembre de 2006.

Loi, M. \& Di Guardo, M. (2015). The third mission of universities: An investigation of the espoused values. Science and Public Policy, 42, 855-870.

Mancinas, R., Romero-Rodríguez, L. \& Aguaded, I. (2016). Problemas de la divulgación de las investigaciones en Comunicación en revistas de alto impacto en español. Revista Faro, Universidad de Playa Ancha, 1, 23, 241-258.

Martínez-Fresneda, H. (2006). "Hacia una nueva teoría de la comunicación". Comunicación y Hombre, 2, 17-18.

Martínez, R. (2015). Nuevos desafíos, nueva reflexión. La nueva teoría estratégica. Razón y Palabra, 91, 721-729.

Massoni, S., Spagnolo, J. \& Gerson, E. (2015). Comunicación estratégica: indicadores comunicacionales en la dimensión interaccional. Mediaciones sociales, 11(14), 88-102.

Merino, L. (2004). El sistema universitario y la vinculación cultural con el medio. Revista Calidad en la Educación, 20, 77-84.

Nothhaft, H. (2016). A Framework for Strategic Communication Research: A Call for Synthesis and Consilience. International Journal of Strategic Communication, 10(2), 6986.

Pérez, R. (2008). Estrategias de Comunicación. Ariel Editores, España, Barcelona, $4^{\text {a }}$ Edición.

Pérez, R. (2012). El estado del arte en la Comunicación Estratégica. Mediaciones Sociales (10), págs. 121-196.

Pérez, R. (2014). La estrategia como campo de estudio. ¿Tenemos ya un nuevo paradigma? Strategy as a field of study. Do we have a new paradigm? Revista Mediterránea de Comunicación, Vol. 5(2), 9-31

Pineda de Alcázar, M. (2006). La investigación de la comunicación en América Latina: Evaluación del estado de la cuestión. Opción, 22(50), 142-158. http://www.scielo.org.ve/scielo.php?script=sci_arttext\&pid=S1012- 


\section{$15872006000200008 \& \operatorname{lng}=$ es\&tlng=es}

Ruff, C. \& Ruiz, M. (2012). Vinculación con el medio, a través de la creación de valor compartido entre la universidad y la sociedad. En: Retos y desafíos de la prospectiva para el desarrollo regional en América Latina, por Mera, C. (editor), Universidad Nacional Abierta y a Distancia.

Salas, C. (2011). Estado del arte de la nueva comunicación estratégica en Iberoamérica y Colombia. Signo y pensamiento (31), págs. 234-246. http://www.scielo.org.co/pdf/signo/v30n59/v30n59a16.pdf

Secundo, G., Pérez, S., Martinaitis, Z. \& Leitner, K. (2017). An Intellectual Capital framework to measure universities' third mission activities. Technological Forecasting \& Social Change, 123, 229-239.

Schubert, T. \& Kroll, H. (2016). Universities' effects on regional GDP and unemployment: The case of Germany. Papers in Regional Science, 95:467-489.

Von Baer, H. (2009). Vinculación con el medio: ¿función subalterna o esencial de la universidad. En: Desafíos y perspectivas de la dirección estratégica de las instituciones universitarias, por Fleet, N. (editor) Santiago de Chile: Comisión Nacional de Acreditación.

https://www.cnachile.cl/Biblioteca\%20Documentos\%20de\%20Interes/DesafiosyPersp ectivas.pdf

\section{Patricia Ferrada Montecinos}

Periodista, Universidad de Concepción. Diplomada en Estrategia y Comunicación Digital y Diplomada en Negociación y liderazgo en escenarios de Conflicto, Universidad UNIACC. Docente de Pedagogía en Lengua Castellana y Comunicación, Universidad Adventista de Chile. Diplomada en coaching y expresión escénica, Universidad de Concepción. Magister en Gestión Estratégica de Comunicación, UNIACC.

Email: patricia.ferrada@gmail.com

\section{Jorge Pinilla Andrade}

Antropólogo, Universidad Austral de Chile. Diplomado en Estrategia y Comunicación Digital y Diplomado Negociación y liderazgo en escenarios de Conflicto, Universidad de las Artes, Ciencias y Comunicación - UNIACC. Diplomado Gestión y puesta en valor de bienes culturales locales para el emprendimiento creativo, artístico y cultural. Universidad de Playa Ancha - UPLA. Diplomado Gestión Pública para el Desarrollo Territorial, FLACSO-OEA. Magister en Gestión Estratégica de Comunicación, UNIACC. Email: j.pinilla.andrade@gmail.com 